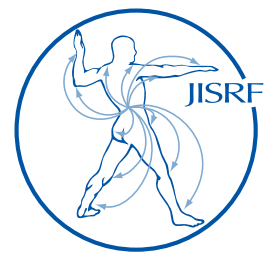

\title{
Tourniquet Application During Total Knee Arthroplasty Does Not Benefit Perioperative Blood Loss or Transfusion Requirement with the Routine Use of Tranexamic Acid
}

Watters $T^{1}$, Levy $D^{1}$, Kim $R^{1}$, Miner $T^{1}$, Dennis $D^{1}$, Jennings $J^{1}$

\section{Abstract}

Background: The use of a tourniquet during total knee arthroplasty (TKA) continues to be a matter of debate. Advantages of tourniquet use include improved visualization, decreased intraoperative and total blood loss, and possibly decreased transfusion requirement. However, the recent widespread adoption of antifibrinolytic therapy with tranexamic acid (TXA), may negate these benefits. The purpose of this study was to evaluate perioperative blood loss and transfusion requirement with two different tourniquet application strategies, and surgery without the use of a tourniquet during routine, primary cemented TKA.

Methods: A retrospective cohort study was performed of 300 patients undergoing TKA at a single institution after the implementation of a routine intravenous TXA administration protocol and consisted of three groups based on tourniquet usage: tourniquet inflation before incision and deflation following cement hardening (TQ), tourniquet inflation prior to cement application and deflation following hardening (Partial TQ), and no tourniquet usage (No TQ). Each group consisted of 100 consecutive patients. Perioperative blood loss, change in hematocrit and transfusion requirement were compared between groups.

Results: Total blood loss (estimated blood loss and drain output) was lowest in the TQ group, however this was only due a slight decrease in intraoperative estimated blood loss. There was no difference in post-operative drain output, or change in hematocrit levels from preoperative values. There were no transfusions in the Partial TQ and No TQ groups, whereas there were 5 transfusions in the TQ group.

Conclusions: In the era of routine TXA administration during TKA, tourniquet usage does not appear to have a benefit in regards to perioperative blood loss or transfusion requirement.

Keywords: tranexamic acid; tourniquet; blood conservation; total knee arthroplasty

Level of Evidence: AAOS Therapeutic Level III

1 Tyler Steven Watters, MD; Daniel L Levy, BS; Raymond H Kim, MD*; Todd M Miner, MD; Douglas A Dennis, MD*; Jason M Jennings, MD, DPT

Colorado Joint Replacement, 2535 S Downing St, Denver, CO 80210 (*Additional affiliations shown on page 47)

(Direct reprint requests to Douglas A Dennis) (c) 2016 Watters, Levy, Kim, Miner, Dennis, Jennings. All rights reserved. Reconstructive Review is a peer-reviewed, open-access orthopaedic journal devoted to oPEN 2 ACCESS
publishing papers in the area of reconstructive arthroplasty. Authors retain copyright

and grant the journal right of first publication with the work. Reconstructive Review follows the Creative Commons Attribution-NonCommercial CC BY-NC. This license allows anyone to download works, build upon the material, and share them with others for non-commercial purposes as long as they credit the senior author, Reconstructive Review, and the Joint Implant Surgery \& Research Foundation (JISRF). 


\section{Introduction}

Tourniquet usage during total knee arthroplasty (TKA) is commonplace among most arthroplasty surgeons in North America. An analysis of practice patterns of members of the American Association of Hip and Knee Surgeons in 2010 found that tourniquet was used in $95 \%$ of patients without vascular disease [1]. Proposed advantages of routine tourniquet usage include improved visualization, decreased intraoperative blood loss, lower transfusion requirement, and possibly superior cementation of components. However, tourniquet usage may be a risk factor for postoperative thromboembolism [2,3], and other wound complications related to tissue hypoxia and reperfusion injury [4,5]. In addition, a recent study found that tourniquet usage may result in diminished quadriceps function during the first 3 months after surgery [6]. Two recent meta-analyses of the available randomized controlled trials (RCTs) on tourniquet usage both concluded that the available evidence supports the assertion that TKA with a tourniquet results in a significant decrease in intraoperative blood loss and transfusion rate, albeit with a slightly higher rate of minor wound complications $[7,8]$. Proponents of tourniquet usage argue that potential disadvantages are outweighed by the benefits of improved visualization and specifically blood conservation, as blood transfusion may increase the risk of postoperative surgical site infection in addition to prolonging length of stay and increasing cost [9].

The recent widespread adoption of routine antifibrinolytic therapy with tranexamic acid (TXA) during total joint arthroplasty has had dramatic effects on blood conservation. TXA is a synthetic drug which inhibits fibrinolysis and clot degradation and may be administered in intravenous (IV), topical, or oral forms. Numerous studies in recent years have highlighted the effectiveness of TXA in decreasing perioperative blood loss and transfusion requirements without significant risk of adverse events or perioperative thromboembolic phenomena [10]. A recent meta-analysis of RCTs evaluating the effectiveness of TXA in primary TKA showed that, when compared to placebo, TXA reduced blood loss by roughly $500 \mathrm{~mL}$ and resulted in 1.43 less units of blood transfused per patient [11].

As such, the purported benefits of decreased blood loss and resultant decreased transfusion requirement with traditional tourniquet use during TKA may no longer be significant in an era where the use of antifibrinolytics during elective knee arthroplasty is routine. The purpose of this study, therefore, was to evaluate perioperative blood loss and transfusion requirement with two different tourniquet application strategies, and surgery without the use of a tourniquet during cemented, primary TKA after the im- plementation of a routine intravenous TXA administration protocol at a single institution using a modern protocol for total joint arthroplasty.

\section{Materials and Methods}

A retrospective cohort study was conducted of 300 patients undergoing primary, cemented TKA after the initiation of a standardized, perioperative IV TXA administration protocol which was implemented at our institution in August 2014. All surgeries were performed by one of four high-volume, fellowship-trained arthroplasty surgeons. During this time period, tourniquet use practices varied among surgeons in our practice: one surgeon routinely used a tourniquet throughout the procedure; one surgeon routinely used a tourniquet only for cementing; and two surgeons did not routinely use a tourniquet at all. Each study group, therefore, consisted of 100 consecutive patients undergoing primary, unilateral TKA based on tourniquet usage: tourniquet inflation before incision and deflation following cement hardening (TQ), tourniquet inflation prior to cement application and deflation following hardening (Partial TQ), and no tourniquet usage (No TQ). Patients were excluded from the study if they had a history of congenital or acquired coagulopathy, liver or renal failure, history of preoperative anti-coagulation, or a contraindication for TXA (ie color blindness, history of stroke) or tourniquet usage (i.e. severe peripheral arterial disease or prior lower extremity bypass). Patients undergoing simultaneous, bilateral TKA and uncemented TKA were also excluded.

TXA was administered on a standardized perioperative regimen of $1 \mathrm{~g}$ IV at the time of skin incision and 1 $\mathrm{g} I \mathrm{~V}$ at the time of closure. In addition to sequential compression devices and early mobilization, DVT prophylaxis regimen included chemoprophylaxis which was initiated on post-operative day 1 . The choice of chemoprophylactic agent was based on risk stratification with the vast majority of patients being treated with aspirin. All procedures were performed under spinal anesthetic with an adductor canal block.

Basic demographic data (including age, sex and BMI) was obtained through our institutional database and individual chart review was subsequently performed on all consecutive patients identified as meeting inclusion criteria. Preoperative hemoglobin $(\mathrm{Hb})$ and hematocrit $(\mathrm{Hct})$ values, and operative time where compared across groups as independent variables. Tourniquet time was recorded for the TQ and Partial TQ groups. Outcome measures included intraoperative estimated blood loss (EBL), post-opera- 
tive hemovac drain output, total blood loss $(\mathrm{EBL}+$ drain output), change in Hct on postoperative day 2 from preoperative values, and transfusion requirement. Descriptive statistics were obtained for demographic data and independent variables, and outcome measures. Group sample distributions were compared using Kruskal-Wallis (median) or Pearson's Chi-squared tests where appropriate. The treatment effect of the 3 study groups was analyzed with respect to the outcome measures using analysis of variance (ANOVA) followed by multiple comparisons using pairwise t-test with Bonferroni correction and Tukey's HSD, or Fisher's exact test where appropriate.

\section{Results}

All surgeries were performed between August 2014 and June 2015. Demographic data for the 3 tourniquet treatment groups is provided in Table 2. The mean age of the Partial TQ group was 67.3 years, which was older than the TQ and No TQ groups (63.0 and 63.6, respectively), and was statistically significant $(\mathrm{p}=0.008)$. The mean BMI of the TQ group was $31.9 \mathrm{~kg} / \mathrm{m} 2$, which was greater than the Partial TQ and No TQ groups (28.4 and 29.8, respective-

Table 1. Proposed Advantages and Disadvantages of Tourniquet Usage in TKA

\begin{tabular}{|l|l|}
\hline Advantages & Disadvantages \\
\hline $\begin{array}{l}\text { Decreased intraoperative blood } \\
\text { loss }\end{array}$ & $\begin{array}{l}\text { Higher rate of deep venous } \\
\text { thrombosis }\end{array}$ \\
\hline Lower transfusion rate & $\begin{array}{l}\text { Increased risk of superficial } \\
\text { wound infection }\end{array}$ \\
\hline Shorter operative time & More pain and swelling \\
\hline $\begin{array}{l}\text { Improved cementation of } \\
\text { components }\end{array}$ & $\begin{array}{l}\text { Decreased quadriceps function } \\
\text { and recovery }\end{array}$ \\
\hline
\end{tabular}

Table 2. Patient Demographics

\begin{tabular}{|l|l|l|l|l|}
\hline & Age & Male (n) & Female (n) & BMI \\
\hline No Tourniquet & $63.8 \dagger$ & 43 & 57 & $29.8 \ddagger$ \\
\hline Partial Tourniquet & $67.3 \dagger$ & 35 & 65 & $28.4 \ddagger$ \\
\hline Tourniquet & $63.0 \dagger$ & 37 & 63 & $31.9 \ddagger$ \\
\hline
\end{tabular}

$\dagger$ - denotes statistical significance between No TQ and TQ versus Partial TQ

₹-denotes statistical significance between No TQ and Partial $T Q$ versus $T Q$

Table 3. Blood Loss Parameters

\begin{tabular}{|l|l|l|l|l|l|l|l|l|}
\hline & Pre-op Hgb & Pre-op Hct & EBL & HV Output & TBL & Post-op Hct & Delta Hct & Transfusions (n) \\
\hline No Tourniquet & 14.8 & 43.3 & $141 \dagger$ & 194 & 334.2 & 32.58 & 10.66 & 0 \\
\hline $\begin{array}{l}\text { Partial } \\
\text { Tourniquet }\end{array}$ & 14.6 & 42.9 & $136.5 \dagger$ & 225.1 & $361.6 \ddagger$ & 32 & 10.93 & 0 \\
\hline Tourniquet & 14.5 & 42.9 & $93.7 \dagger$ & 188.3 & $282 \ddagger$ & 32.42 & 10.53 & 5 \\
\hline
\end{tabular}

$\dagger$ - denotes statistical significance between No TQ and Partial TQ versus TQ

$\mp$-denotes statistical significance between $T Q$ and Partial TQ

ReconstructiveReview.org • JISRF.org • Joint Implant Surgery \& Research Foundation ly), and was statistically significant ( $\mathrm{p}=0.002)$. There was no statistically significant difference between the number of male and female patients in each group.

Operative time was slightly longer in the No TQ group (67.1 minutes), compared to the Partial TQ and TQ groups (63.2 and 64.5, respectively), and was also statistically significant $(\mathrm{p}=0.009)$. The average tourniquet time in the Partial TQ group, where the tourniquet was only inflated for cementing of the prosthesis, was 12.6 minutes. The average tourniquet time in the TQ group was 54.1 minutes.

Preoperative $\mathrm{Hb}$ and Hct values were similar among the 3 treatment groups (Table 2). EBL was higher in the No TQ group (141.0 mL) and Partial TQ $(136.5 \mathrm{~mL})$ than in the TQ group $(93.7 \mathrm{~mL})$. However, there was no difference in postoperative hemovac drain output between groups (ANOVA $\mathrm{p}=0.198$ ). The significant differences in EBL translated to differences seen between groups in TBL. However, ANOVA testing with multiple comparison tests revealed that the only significant difference in TBL was between TQ and Partial TQ.

The differences between the groups in EBL and TBL did not appear to translate to a clinical difference between the groups. Postoperative Hct was similar for all 3 groups and, likewise, there was no difference between the treatment groups with respect to change in Hct from preoperative values (ANOVA $p=0.733$ ). Furthermore, there were no transfusions in either the No TQ or Partial TQ groups, whereas 5 patients in the TQ group required blood transfusion post-operatively. The average number of units transfused per TQ patient was 1.8.

\section{Discussion}

Tourniquet usage is common amongst arthroplasty surgeons during TKA. From a subjective standpoint, the use of a tourniquet provides a bloodless surgical field and optimizes visualization during the procedure [9]. A recent meta-analysis of the available RCTs comparing TKA with and without use of a tourniquet was performed by Jiang et al [8]. Their results demonstrated that TKA with use of a tourniquet has decreased intraoperative blood loss, transfusion rate, and operative time, but also appeared to result 
in slower early functional recovery with an increased rate of deep venous thrombosis and minor wound complications. However, of the 26 independent RCTs included in the overall meta-analysis, only 5 had been published since 2010. The purpose of our study was to evaluate blood loss and transfusion requirement with and without tourniquet after implementation of a contemporary blood conservation protocol utilizing IV TXA.

While there are more historical studies documenting the efficacy of TXA in reducing blood loss and transfusion requirements $[12,13]$, the literature on the subject has increased dramatically over the past few years [10], as the use of TXA has become nearly universal at centers throughout North America. Studies have shown a low risk of thromboembolic complications with TXA use, even with less aggressive chemoprophylactic regimens using aspirin [14]. In a large retrospective cohort study of more than 13,000 elective hip and knee replacement patients at a single center, Duncan et al. showed that the odds of post-operative VTE and 30-day mortality were unchanged with IV TXA administration [15]. Efficacy in terms of blood loss and reduction in transfusion requirement has also been shown with topical administration during TKA in several recent RCTs [16,17].

The results of our study suggest that, in the setting of routine intraoperative anti-fibrinolytic administration, while perioperative blood loss may be lower with use of a tourniquet, the difference is relatively small and may be clinically negligible. There was no difference between the TQ, Partial TQ, and No TQ groups with respect to postoperative drain output or change in hematocrit values from preoperative levels (Table 2). Moreover, there were no transfusions in the Partial TQ and No TQ groups postoperatively. There were 5 transfusions in the TQ group, despite this group having on average the lowest quantitative perioperative blood loss. Because all of these patients were treated by a single surgeon, this may represent a selection bias based on a lower threshold for post-operative transfusion by the treating surgeon. At our institution, the decision to transfuse blood products to a patient cannot be standardized and remains a clinical decision requiring informed consent based on a diagnosis of symptomatic anemia requiring transfusion made by the treatment team. Nonetheless, no patients that underwent TKA without tourniquet (No TQ), or that underwent TKA with tourniquet inflation for cementing only (Partial TQ) were transfused in this large series of consecutive patients, despite the fact that these two groups had greater intraoperative blood loss and would theoretically be at higher risk for transfusion.

While tourniquet use during TKA is clearly a historical norm amongst arthroplasty surgeons, recent attention has been given to the potential detrimental functional effects that result from its routine use. In a prospective randomized study of 28 patients undergoing same-day bilateral TKA where tourniquet was used throughout the procedure on one limb and not on the contralateral, Dennis et al. found lower post-operative quadriceps strength on the limb with the tourniquet that persisted at 3 months after surgery [6]. A recent study by Huang et al, comparing 3 different tourniquet application strategies using a "mini-midvastus" approach, found that serum inflammatory and muscle injury markers were lowest in the group where tourniquet was used only for cementation and not for the entire procedure [18]. Another recent randomized trial evaluating tourniquet use only for cementation during TKA found no difference in terms of surgical time, hemoglobin change or total blood loss compared to prolonged tourniquet use and reported one case of compartment syndrome in a patient that had tourniquet inflation until closure [19]. The authors of that study suggested that tourniquet inflation for cementation only may be beneficial for providing a bloodless field during implant fixation without the risks of prolonged tourniquet use. However, a recent prospective RCT using radioisometric analysis at 2 years post-op found no difference in tibial component migration between cemented knees done with and without a tourniquet, suggesting the surgery without the use of a tourniquet does not influence implant fixation in the short-term [20].

Limitations of this study include those inherent with a retrospective study design. We concede that the methods used to assess blood loss intraoperatively and postoperative may lack precision as a result of factors such as blood loss on drapes, plugged postoperative drainage systems, etc. We believe reporting the change in preoperative to postoperative hematocrit change is more accurate and therefore report these findings as well. In addition, surgeries were performed by one of four surgeons, and therefore minor variations in surgical technique may have some influence on perioperative blood loss. Lastly, as stated above there may be a selection bias with regards to the threshold for transfusion by the surgeon who routinely used the tourniquet, as the decision to transfuse a patient is not standardized at our institution and is based on the clinical judgement of the treating physician based on a diagnosis of symptomatic anemia requiring transfusion and requiring informed patient consent. Further research is justified to see if a more standardized blood product utilization protocol would improve our blood conservation program further. 


\section{Conclusion}

In the era of improved perioperative blood conservation methods with spinal anesthesia and anti-fibrinolytic therapy using TXA, prolonged tourniquet use during TKA does not appear to have a significant clinical impact on perioperative blood loss and transfusion requirement, and may not be justified based on known higher rates of wound complications and slowed functional recovery.

\section{Disclosure}

The authors declare that there is no conflict of interest regarding the publication of this paper. For full disclosures refer to last page of this journal.

Additional Affiliations Raymond H Kim, MD

- Adjunct Professor of Bioengineering, Department of Mechanical and Materials Engineering, University of Denver, Denver, CO

- Clinical Associate Professor, Department of Orthopaedic Surgery, Joan C. Edwards School of Medicine at Marshall University, Huntington, WV

Additional Affiliations Douglas A Dennis, MD

- Adjunct Professor, Department of Biomedical Engineering, University of Tennessee, Knoxville, TN

- Adjunct Professor of Bioengineering, Department of Mechanical and Materials Engineering, University of Denver, Denver, CO

- Assistant Clinical Professor, Department of Orthopaedics, University of Colorado School of Medicine, Denver, CO

\section{References}

1. Berry DJ, Bozic KJ. Current practice patterns in primary hip and knee arthroplasty among members of the American Association of Hip and Knee Surgeons. J Arthroplasty. 2010;25:2-4.

2. Wauke K, Nagashima M, Kato N, Ogawa R, Yoshino S. Comparative study between thromboembolism and total knee arthroplasty with or without tourniquet in rheumatoid arthritis patients. Arch Orthop Trauma Surg. 2002;122:442-6.

3. Kato N, Nakanishi K, Yoshino S, Ogawa R. Abnormal echogenic findings detected by transesophageal echocardiography and cardiorespiratory impairment during total knee arthroplasty tourniquet. Anesthesiology. 2002;97:1123-8.

4. Abdel-Salam A, Eyres, KS. Effects of tourniquet during total knee arthroplasty. A prospective randomized study. J Bone Joint Surg Br. 1995:77:250-3.

5. Clarke MT, Longstaff L, Edwards D, Rushton N. Tourniquet induced wound hypoxia after total knee replacement. J Bone Joint Surg Br. 2001;83:40-4.
6. Dennis DA, Kittleson AJ, Yang CC, Miner TM, Kim RH, Stevens-Lapsley JE. Does tourniquet use in TKA affect recovery of lower extremity strength and function? A randomized trial. Clin Orthop Relat Res. 2016;474(1):69-77.

7. Alcelik I, Pollock RD, Sukeik M, Bettany-Saltikov J, Armstrong PM, Fismer P. A comparison of outcomes with and without a tourniquet in total knee arthroplasty: A systemative review and meta-analysis of the randomized controlled trials. J Arthroplasty. 2012;27:331-40.

8. Jiang F, Zhong H, Hong Y, Zhao G. Use of a tourniquet in total knee arthroplasty: a systematic review and meta-analysis of randomized controlled trials. J Orthop Sci. 2015;20:110-23.

9. Parvizi J, Diaz-Ledezma C. Total knee replacement with the use of a tourniquet: more pros than cons. Bone Joint J. 2013;95-B, Supple A:133-4.

10. Melvin JS, Stryker LS, Sierra RJ. Tranexamic acid in hip and knee arthroplasty. J Am Acad Orthop Surg 2015;23:732-40.

11. Yang ZG, Chen WP, Wu LD. Effectiveness and safety of tranexamic acid in reducing blood loss in total knee arthroplasty: A meta-analysis. J Bone Joint Surg Am. 2012;94:1153-9.

12. Benoni G, Fredin H. Fibrinolytic inhibition with tranexamic acid reduces blood loss and blood transfusion after knee arthroplasty: A prospective, randomized, double blind study of 86 patients. J Bone Join Surg Br 1996;78:434-40.

13. Tanaka N, Sakahashi H, Sato E, Hirose K, Ishima T, Ishii S. Timing of the administration of tranexamic acid for maximum reduction in blood loss in arthroplasty of the knee. J Bone Joint Surg Br 2001;83:702-5.

14. Gillette BP, DeSimone LJ, Trousdale RT, Pagnano MW, Sierra RJ. Low risk of thromboembolic complications with tranexamic acid after primary total hip and knee arthroplasty. Clin Orthop Relat Res. 2013;471:150-4.

15. Duncan CM, Gillette BP, Jacob AK, Sierra RJ, Sanchez-Sotelo J, Smith HM. Venous thromboembolism and mortality associated with tranexamic acid use during total hip and knee arthroplasty. J Arthroplasty. 2015:30:272-276.

16. Gomez-Barrena E, Ortega-Andreu M, Padilla-Eguiluz NG, Perez-Chrzanowska H, Figueredo-Zalve R. Topical intra-articular compared with intravenous tranexamic acid to reduce blood loss in primary total knee replacement: a doubleblind, randomized, controlled, noninferiority clinical trial. J Bone Joint Surg Am. 2014;96:1937-44.

17. Aguilera X, Martinez-Zapata MJ, Hinarejos P, Jordan M, Leal J, Gonzalez JC, Monllau JC, Celaya F, Rodriquez-Arias A, Fernandez JA, Pelfort X, Puig-Verdie LI. Topical and intravenous tranexamic acid reduce bloos loss compared to routine hemostasis in total knee arthroplasty: a multicenter, randomized, controlled trial. Arch Orthop Trauma Surg. 2015:135:1017-25.

18. Huang ZY, Pei FX, Ma J, Yang J, Zhou ZK, Kang PD, Shen B. Comparison of three different tourniquet application strategies for minimally infasive total knee arthroplasty: a prospective non-randomized clinical trial. Arth Orthop Trauma Surg. 2014;134:561-70.

19. Tarwala R, Dorr LD, Gilbert PK, Wan Z, Long WT. Tourniquet use during cementation only during total knee arthroplasty: a randomized trial. Clin Orthop Relat Res. 2014;472:169-74.

20. Ejaz A, Laursen AC, Jakobsen T, Rasmussen S, Nielsen PT, Laursen MB. Absence of a tourniquet does not affect fixation of cemented TKA: a randomized RSA study of 70 patients. J Arthroplasty. 2015;30:2128-32. 